\title{
Anuloplastia Posterior a Céu Fechado: Promessa ou Realidade?
}

\author{
Ver artigo relacionado \\ na página 183
}

\author{
Carlos Manuel de Almeida Brandão', \\ Pablo Maria Alberto Pomerantzeff ${ }^{1}$
}

A importância da insuficiência cardíaca congestiva tem sido subestimada nos dias atuais, apesar da elevada prevalência dessa doença e de seu prognóstico desfavorável ${ }^{1}$. Para se ter uma ideia desse fato, cerca de 4,7 milhões de pessoas nos Estados Unidos são portadoras de insuficiência cardíaca, resultando em mais de 1 milhão de internações por ano, sendo a principal causa de hospitalização em pacientes com idade superior a 65 anos. Com o aumento da expectativa de vida das pessoas em todo o mundo e a melhora da qualidade de assistência médica prestada, com consequente redução da mortalidade por infarto agudo do miocárdio e outras doenças cardíacas, esperase uma incidência cada vez maior de insuficiência cardíaca no futuro ${ }^{2}$.

O tratamento cirúrgico de eleição para a insuficiência cardíaca em fase avançada é o transplante cardíaco; porém, em decorrência do limitado número de doadores e dos pacientes com contraindicações ao transplante, como idade, doenças associadas e hipertensão pulmonar, entre outras, o tratamento cirúrgico da valva mitral pode ser uma alternativa ${ }^{3}$.

A substituição da valva mitral está relacionada à piora da função ventricular sistólica, em decorrência da perda de parte da continuidade anel-cordas tendíneas-papilares, o que está associado a maior mortalidade hospitalar e a pior sobrevida tardia ${ }^{4}$.

A plástica da valva mitral, por preservar o aparelho subvalvar mitral, apresenta baixa mortalidade hospitalar, boa sobrevida tardia, melhor função ventricular, menor estresse sistólico e volume ventricular esquerdo ${ }^{5}$. Bolling et al. ${ }^{6}$ propuseram anuloplastia mitral com anel flexível para a correção da insuficiência mitral na cardiomiopatia terminal, apresentando baixa mortalidade hospitalar e sobrevida em 40 meses de $71 \%$. Chen et al. ${ }^{7}$, em série de 81 pacientes com insuficiência mitral e fração de ejeção inferior a 30\%, demonstraram aumento significativo da fração de ejeção e da classe funcional após a plástica da valva mitral, com sobrevida tardia de 38\% em cinco anos. Os fatores de risco para a mortalidade foram o grau de insuficiência cardíaca e a idade. De acordo com Bolling et al. ${ }^{8}$, a plástica da valva mitral apresenta mortalidade de 5\%, na experiência da Universidade de Michigan, com 92 casos operados entre 1993 e 1999.

Nesta edição da Revista Brasileira de Cardiologia Invasiva, Merino et al. ${ }^{9}$ descrevem a primeira série em humanos com um dispositivo de anuloplastia percutânea (Mitralign), que permite a realização da plástica mitral a céu fechado. No nosso ponto de vista, essa técnica deve ser realizada em caráter de exceção, uma vez que ela não realiza uma anuloplastia completa, ou seja, que trata o anel mitral de trígono a trígono, ponto fundamental para a obtenção de bons resultados a médio e longo $\operatorname{prazos}^{10}$.

Outro ponto que também chama a atenção para a técnica é o grau de redução do anel mitral obtido com essa sutura, uma vez que os autores não quantificaram a área valvar mitral obtida no pós-operatório, citando apenas redução do grau de insuficiência mitral encontrada ao ecocardiograma9. Existe também a possibilidade de distorção das cordas tendíneas com a passagem desses pontos no anel mitral posterior, uma vez que o procedimento é realizado a céu fechado.

Os autores apresentam, nesse artigo original, uma técnica inovadora para o tratamento de uma doença de alta prevalência, mas que deve ser realizada em caráter excepcional, em protocolos especiais, com controle rigoroso dos resultados a médio e a longo prazos, que nos trará resultados mais consistentes ${ }^{9}$.

\footnotetext{
1 Instituto do Coração do Hospital das Clínicas da Faculdade de Medicina da Universidade de São Paulo - São Paulo, SP, Brasil. Correspondência: Carlos Manuel de Almeida Brandão. Av. Dr. Enéas Carvalho de Aguiar, $44-2 \div$ andar - bloco $2-$ Cerqueira César - São Paulo, SP, Brasil - CEP 05403-000

E-mail: dcibrandao@incor.usp.br

Recebido em: 22/6/2009 - Aceito em: 23/6/2009
} 


\section{CONFLITO DE INTERESSES}

Os autores declararam inexistência de conflito de interesses.

\section{REFERÊNCIAS BIBLIOGRÁFICAS}

1. American College of Cardiology/American Heart Association. Guidelines for the evaluation and management of heart failure. Report of the American College of Cardiology/American Heart Association Task Force on Practice Guidelines (Committee on Evaluation and Management of Heart Failure). J Am Coll Cardiol. 1995;26(5):1376-98.

2. Garg R, Packer M, Pitt B, Yusuf S. Heart failure in the 1990s: evolution of a major public health problem in cardiovascular medicine. J Am Coll Cardiol. 1993;22(4 Suppl A):3A-5A.

3. Buffolo E, Paula IA, Palma H, Branco JN. Nova abordagem cirúrgica para o tratamento de pacientes em insuficiência cardíaca refratária com miocardiopatia dilatada e insuficiência mitral secundária. Arq Bras Cardiol. 2000;74(2):129-34.

4. Westaby S. Preservation of left ventricular function in mitral valve surgery. Heart. 1996;75(4):326-9.
5. Pomerantzeff $P M A$, Brandão $C M A$, Faber $C N$, Fonseca $M H$, Puig LB, Grinberg M, et al. Plástica da valva mitral: resultados aos 17 anos de experiência. Rev Bras Cir Cardiovasc. 1999; 14(3):185-90.

6. Bolling SF, Deeb M, Brunsting LA, Bach DS. Early outcome of mitral valve reconstruction in patients with end-stage cardiomyopathy. J Thorac Cardiovasc Surg. 1995;109(4): 676-83.

7. Chen FY, Adams DH, Aranki SF, Collins JJ Jr, Couper GS, Rizzo RJ, et al. Mitral valve repair in cardiomyopathy. Circulation. 1998;98(19 Suppl):II124-7.

8. Bolling SF, Dickstein ML, Levy JH, McCarthy PM, Oz MC, Savage RM. Management strategies for high-risk cardiac surgery: improving outcomes in patients with heart failure. Heart Surg Forum. 2000;3(4):337-49.

9. Merino SFG, Ebner A, Alvarez E, Silva E. Mitralign percutaneous mitral annuloplasty system for mitral regurgitation in $\mathrm{CHF}$ patients. First in man experience. Rev Bras Cardiol Invas. 2009;17(2):183-9.

10. Carpentier A. Cardiac valve surgery: the "French correction". J Thorac Cardiovasc Surg. 1983;86(3):323-37. 